\title{
Cutaneous Phaeohyphomycosis Caused by Exophiala attenuata in a Domestic Cat
}

\author{
David P. Overy $\cdot$ Chelsea Martin • \\ Anne Muckle $\cdot$ Lorraine Lund • \\ Jill Wood · Paul Hanna
}

Received: 16 March 2015/Accepted: 10 June 2015/Published online: 19 June 2015

(C) Springer Science+Business Media Dordrecht 2015

\begin{abstract}
A 7-year-old female-spayed, domestic short-haired cat was presented to her veterinarian with a mass on the hind paw. Histopathologic examination of a tissue biopsy revealed nodular pyogranulomatous panniculitis with intralesional pigmented fungal hyphae. A dematiaceous fungal isolate was isolated with a micromorphological phenotype consistent with the anamorphic genus Exophiala: budding cells, torulose mycelium and annellidic
\end{abstract}

D. P. Overy · C. Martin · A. Muckle · P. Hanna

Department of Pathology and Microbiology, Atlantic Veterinary College, University of Prince Edward Island, 550 University Avenue, Charlottetown, PE C1A 4P3, Canada

D. P. Overy

Department of Chemistry, University of Prince Edward Island, 550 University Avenue, Charlottetown,

PE C1A 4P3, Canada

D. P. Overy $(\bowtie)$

Nautilus Biosciences Canada Inc., Duffy Research Center, 550 University Avenue, Charlottetown, PE C1A 4P3, Canada

e-mail: dovery@upei.ca

A. Muckle · L. Lund

Diagnostic Services, Atlantic Veterinary College, University of Prince Edward Island, 550 University Avenue, Charlottetown, PE C1A 4P3, Canada

J. Wood

Southport Animal Hospital, 23 Stratford Road, Stratford, PE C1B 1T4, Canada conidiogenesis from simple conidiophores consisting of terminal and lateral cells that tapered to a short beak at the apex. Sequence homology of the internal transcribed spacer region of the rDNA gene confirmed the identification of the isolate as Exophiala attenuata. Reported here is the first confirmed case of feline phaeohyphomycosis caused by E. attenuata in North America. Similar to historical cases of feline phaeohyphomycosis caused by Exophiala spp., there was no history or postmortem evidence to suggest the patient was in an immunocompromised state (e.g., suffering from FeLV or FIV). Although aggressive surgical excision of local lesions is recommended prior to drug treatment when dealing with subcutaneous phaeohyphomycosis, surgery followed by itraconazole treatment did not resolve the E. attenuata infection in this cat.

Keywords Phaeohyphomycosis $\cdot$ Felis catus $\cdot$ Black yeast $\cdot$ Exophiala attenuata $\cdot$ Molecular barcoding

\section{Introduction}

Melanized (dematiaceous) fungi known as "black yeasts" can cause significant and re-occurring phaeohyphomycosis, chromoblastomycosis and mycetoma in the skin and soft tissues of animals and humans. Deposition of melanin in the fungal cell wall plays an important role in virulence and pathogenicity of these pathogens, aiding to elude host immune responses by 
negating hydrolytic enzymatic attack and scavenging of free radicals liberated by phagocytic cells during the oxidative burst [1]. Black yeasts exist either as a yeastlike state with division by unicellular budding or can grow filamentously. All species known to cause disease are classified in the ascomycete order Chaetothyriales [1], where the majority of the clinically relevant species fall within the Chaetothyriales and belong to the family Herpotrichiellaceae. The ability to reproduce by unicellular growth is found exclusively in the genus Exophiala; for this reason, the majority of disseminated black yeast infections are caused by Exophiala spp. [1]. Aside from causing opportunistic superficial and systemic mycoses in warm- and cold-blooded animals, Exophiala spp. are considered ubiquitous fungi, historically isolated from a variety of environmental substrates such as soil and sediment, decaying wood and plant material, and drinking water [2-4]. Conclusive diagnosis of phaeohyphomycosis caused by an Exophiala spp. requires several steps including histopathologic examination of clinical samples, culture followed by microscopical examination and sequencing of a representative barcoding gene, such as the internal transcribed spacer region or the D1/D2 region of the nuclear large subunit rDNA gene [1, 2, 5]. This case report details the conclusive diagnosis of a phaeohyphomycosis caused by $E$. attenuata occurring in the hind paw of a domestic short haired cat.

\section{Case Description}

A 7-year-old female-spayed, domestic short-haired cat was presented for veterinary evaluation with a mass and fistulous tract on the plantar surface of the right hind paw, immediately proximal to the metatarsal foot pad. The slowly progressive mass was first noticed by the cat's owner approximately 8 months prior to presentation. A tentative diagnosis of chronic inflammation was made, and antibiotic therapy and topical steroids were initiated (injectable cefovecin and topical hydrocortisone). The condition did not respond and the therapy, was discontinued; 2 weeks later, a surgical biopsy was performed to remove the mass. Histopathologic examination revealed an incompletely excised nodular pyogranulomatous dermatitis and panniculitis with intralesional pigmented fungal hyphae (phaeohyphomycosis). Itraconazole oral suspension (10 mg/kg once daily) was started, and the patient initially did well postoperatively, but 6 weeks later her owner reported that the cat had become lethargic, the mass had returned and a new skin mass had developed. The draining, primary mass was swabbed and submitted for culture and fungal identification. The cat was euthanized 2 weeks later due to progressive lethargy and anorexia and development of a distended abdomen. The cat's medical history prior to the development of phaeohyphomycosis was unremarkable. She was regularly vaccinated against feline leukemia virus (FeLV); however, she had not been tested for FeLV or feline immunodeficiency virus (FIV).

\section{Pathology}

Postmortem examination revealed similar microscopic features in the recurrent mass compared to the prior biopsy (Fig. 1); in addition, two smaller cutaneous masses were observed; one located on the anterior surface of the right forelimb, proximal to the carpus, and one located on the plantar surface of the left hind foot, approximately $1 \mathrm{~cm}$ proximal to the metatarsal foot pad. There was no evidence of systemic mycosis; however, peritoneal and pleural effusions were observed, along with subacute centrilobular hepatic congestion with scattered hepatocyte necrosis, acute moderate pulmonary congestion and edema, and mild multifocal subendocardial and
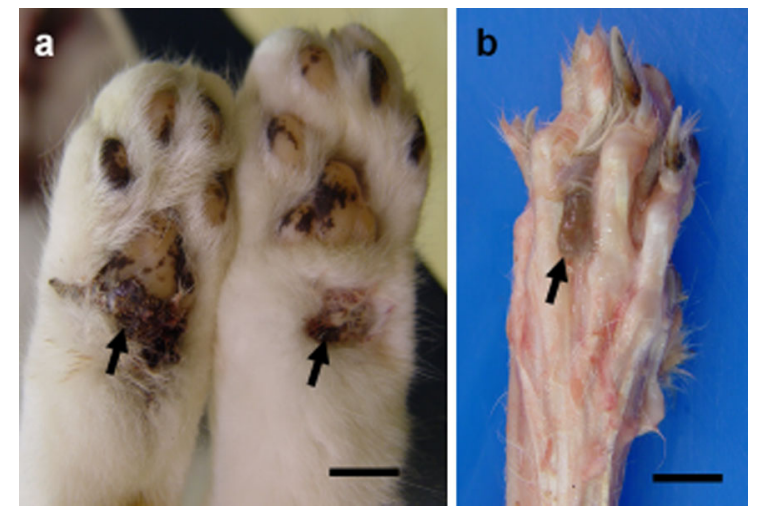

Fig. 1 Gross pathology of cutaneous mycetomas of the hind limbs: a pyogranulomatous and ulcerative dermatitis and cellulitis of the right and left hind paws (black arrows); b dorsal extension of the pigmented pyogranuloma between the second and third digits of the right hind paw (scale bar $1 \mathrm{~cm}$ ) 
myocardial fibrosis. Feline coronavirus (feline infectious peritonitis, FIP) was not detected in the peritoneal effusion.

The microscopic sections from the submitted biopsy of the panniculitis showed large nodular aggregates of inflammatory cells, which were expanding and effacing the subcutis, and occasionally extended into the overlying dermis. The inflammatory cells were predominately epithelioid macrophages with variable numbers of neutrophils, small numbers of lymphocytes and plasma cells, and occasional scattered inflammatory giant cells. Numerous segments of fungal hyphae were scattered throughout the inflammation, which stained very light yellow-brown with hematoxylin and eosin, but stained strongly with both periodic acid Schiff and Gomori's methenamine special stains. The hyphae also stained strongly positive for melanin pigment with Fontana-Masson special stain (Fig. 2). The hyphae were frequently septate, generally narrow $(\sim 2-3 \mu \mathrm{m}$ wide $)$ with occasional torulose expansions (up to $10 \mu \mathrm{m}$ diameter) and had infrequent right-angle branching.

\section{Mycology Findings}

A dematiaceous fungal isolate (denoted as F10685) was obtained from the cutaneous mass excised from the right hind limb. The morphological phenotype of isolate F10685 was examined and compared with the published description of E. attenuata [6]. Colonies

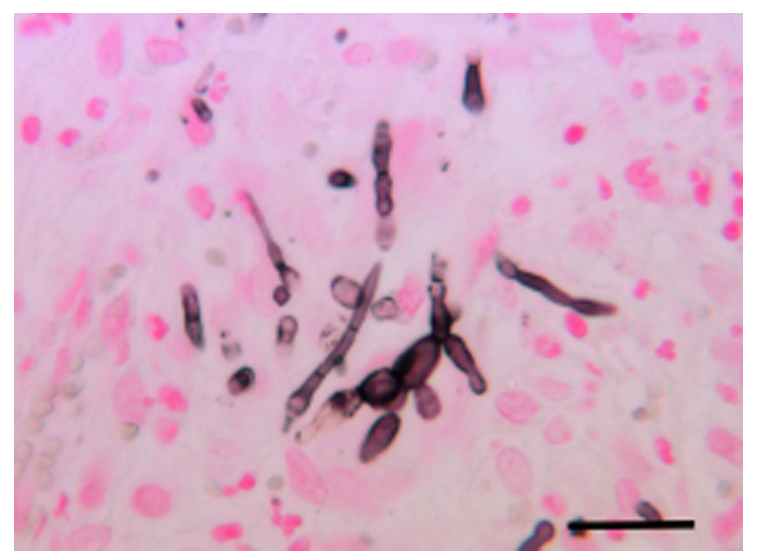

Fig. 2 Melanized, septate and torulose hyphal growth in biopsied tissue as visualized by Fontana-Masson staining (scale bar $25 \mu \mathrm{m})$ were slow growing, olivaceous black and velvety in appearance with a black reverse. Budding cells were present (Fig. 3a, b) and more pronounced in older colonies. Conidia were single celled and variable in shape, ranging from obovoid to narrowly ellipsoidal (Fig. 3c). Conidiogenesis was annellidic with conidia accumulating in slimy masses from intercalary (Fig. 3d) conidiogenous cells or from conidiophores that consisted of terminal and lateral cells tapering to a short beak at the apex and septate at the base (Fig. 3e). Torulose mycelium was also abundant (Fig. 3f). Mature hyphae, conidiophores and conidia were dematiaceous.

Both the ITS rDNA gene and the D1/D2 variable region of the nLSU rDNA gene from F10685 were amplified from gDNA and sequenced (sequences were accessioned into GenBank as KT013095 and KT013094 respectively). Blastn ITS rDNA sequence searches against GenBank returned a $99 \%$ sequence homology (having only 2-5 nucleotide differences) of the isolate F10685 ITS gene with various isolates of $E$. attenuata. A dataset of the ITS rDNA gene was compiled and analyzed to infer the relative evolutionary history of isolate F10685 with other representative Exophiala spp (Fig. 4). The analysis involved 35 sequences and included 549 positions in the final dataset with an overall mean distance calculated as 0.179 with a standard error of 0.017 . Isolate F10685 formed a well-supported clade $(100 \%$ bootstrap support) with other isolates of E. attenuata.

\section{Discussion}

Although micromorphological identification is possible for some Exophiala spp., most taxa can only be recognized with sufficient certainty by using molecular methods, with the ITS rDNA gene having proven to be reliable for species distinction [5]. Micromorphologically, E. attenuata is most similar to E. spinifera as these two species are the only Exophiala spp. that produce large, erect and well-differentiated conidiophores [6]. Although morphologically similar, these two species, however, are readily distinguished by molecular methods using the ITS rDNA gene as demonstrated in Fig. 3. Infections caused by E. attenuata are considered to be rare $(1.6 \%$ overall in an evaluation of human and animal mycoses of Exophiala spp. based on molecular identification [5]) and limited 

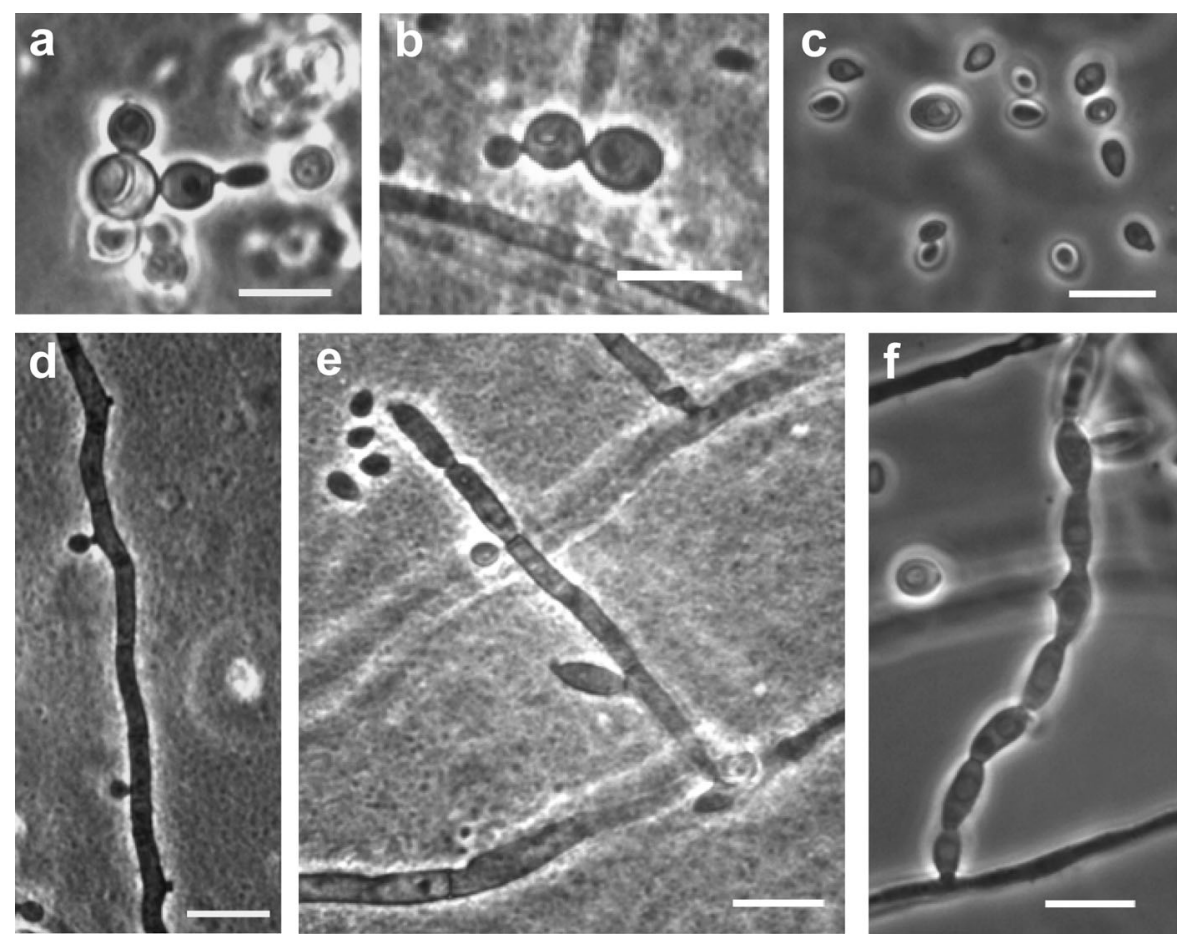

Fig. 3 Micromorphology of Exophiala attenuata (isolate F10685): a, b yeast state demonstrating budding with distinct scarification, often forming long chains; $\mathbf{c}$ conidia single celled and obovoid; $\mathbf{d}$ conidiophores and $\mathbf{e}$ intercalary conidiogenesis observed; f torulose hyphae was abundant (scale bar $10 \mu \mathrm{m}$ ) to phaeohyphomycoses of cutaneous and subcutaneous tissue. Systemic dissemination of E. attenuata is seldom reported, although such mycoses are common for other species of Exophiala, such as E. dermatitidis, E. oligosperma, E. phaeomuriformis, E. xenobiotica and E. lecanii-corni [5].

This case report represents the first report of E. attenuata causing feline phaeohyphomycosis in North America. Phaeohyphomycoses in cats are predominantly subcutaneous, whereas infection of the eyes, bones, central nervous system and organs are rare. Subcutaneous infections observed in cats mainly occur via skin trauma due to puncture wounds often caused by wood [7]. Presumably infection of the cat in this case report occurred in a similar manner via implantation of the pathogen into the subcutaneous tissue of the paw following trauma. Although several fungal species can cause phaeohyphomycoses, previous case reports of feline phaeohyphomycoses caused by Exophiala spp. have been limited to two species, E. jeanselmei and E. spinifera [8-10]. Phylogenetic analysis performed on the Exophiala genus during the description of the
E. attenuata taxon [6] revealed that one of the E. spinifera isolates causing a nasal phaeohyphomycosis in a cat was in fact an isolate of E. attenuata as the isolate conclusively grouped together within the $E$. attenuata clade. Of the remaining two other incidents of phaeohyphomycosis attributed to E. spinifera [9], the identity of only one of these isolates was subsequently confirmed by ITS sequencing [6]. It is possible that the second E. spinifera isolate may have been E. attenuata based on the shared similarities in micromorphology of these two taxa, and also given that the diagnosis in early case reports was made based solely on micromorphology, prior to the description of the E. attenuata taxon. Unfortunately, no voucher specimen was persevered for this isolate; therefore, it is impossible to test this hypothesis using molecular methods. With the addition of our case report, the occurrence of phaeohyphomycosis in cats caused by E. attenuata appears to be more prevalent that previously reported.

In fungal infection cases, one might assume that the patient would have a predisposed susceptibility for infection as a result of an immunocompromised state, 


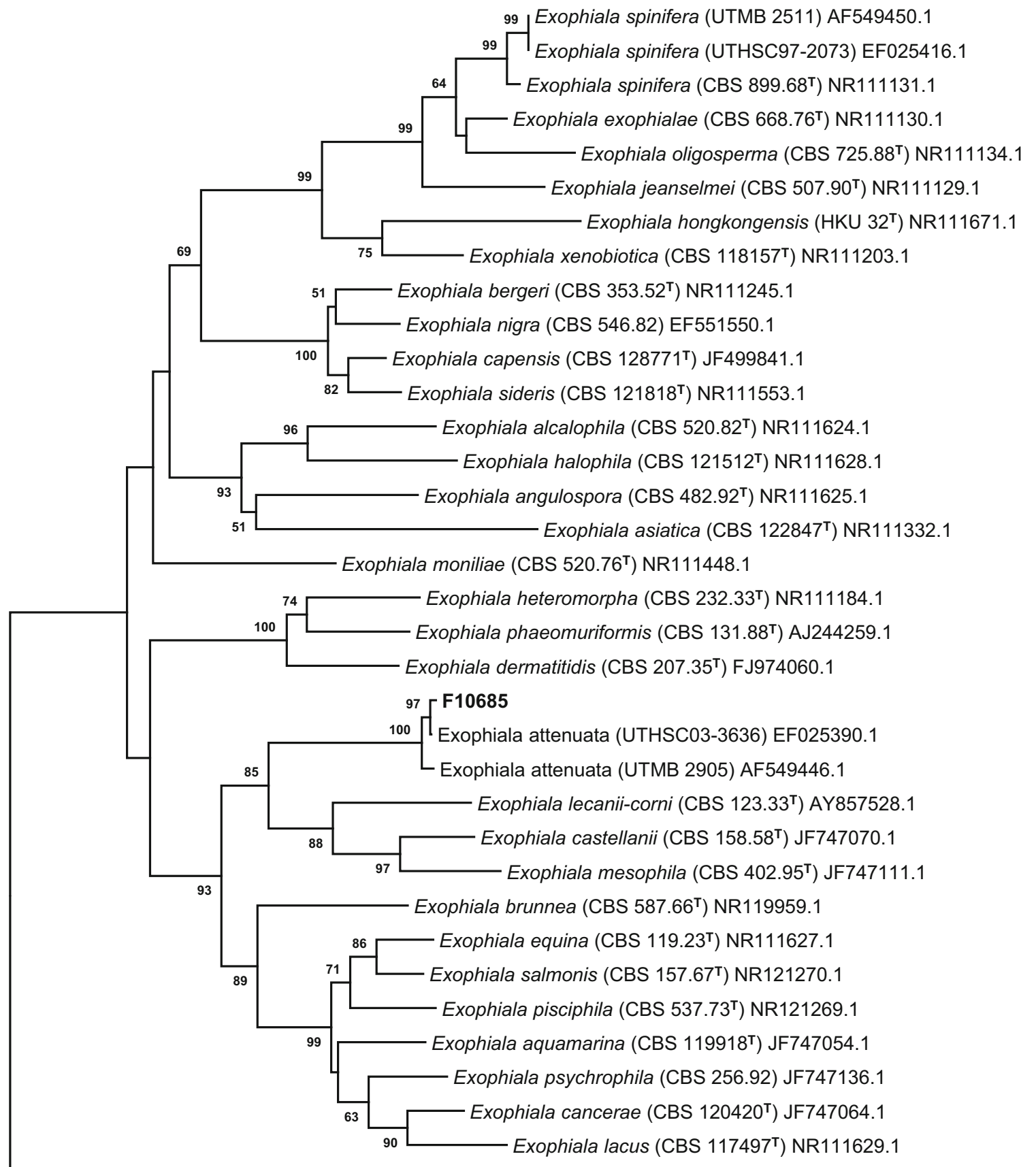

Ceramothyrium melastoma (CBS 133576) KC005771.1

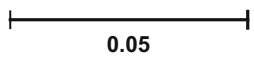

Fig. 4 Bootstrap consensus tree inferred from 2000 replicates using the neighbor-joining method based on Exophiala spp. ITS rDNA sequences (type strains designated as ${ }^{\mathrm{T}}$ ); the percentage of replicate trees $(>50 \%)$ in which the associated taxa clustered together in the bootstrap tests of 2000 replicates are shown next to the branches; evolutionary distances were computed using the maximum composite likelihood method and are in the units of the number of base substitutions per site; the tree was rooted with Ceramothyrium melastoma (CBS 133576) 
possibly caused by a predisposing disease or metabolic factor. In this case, the patient had not been tested for FeLV or FIV infection, and thus, infection with either of these immunosuppressive viruses cannot be entirely ruled out; however, there was no history or postmortem evidence to suggest the patient was suffering from FeLV or FIV. Similar presumptions of a predisposing immunodeficiency were also made in previous case reports of feline phaeohyphomycosis involving E. spinifera, yet in both cases tests for FeLV were found to be negative [9]. In previous reports of phaeohyphomycosis involving E. jeanselmei, there was also no predisposing immunocompromised state determined [10]. In the only other case report of feline phaeohyphomycosis involving E. attenuata (at the time identified as E. spinfera), no predisposing factors could be found and all tests for FIV, FeLV and FIP were negative [8]. Cumulative evidence suggests that healthy cats are susceptible to infection caused by Exophiala spp. and a predisposing immunocompromised state should not be assumed.

Although Exophiala spp. are sensitive to various antifungal drugs when tested in vitro, the outcome of treatment with these drugs in vivo is often poor [11]. Recommendations for the most effective treatment of phaeohyphomycosis require knowledge of the pathogen identity, as drug susceptibility varies between species. In many cases involving Exophiala phaeohyphomycosis, a correlation between MIC values obtained in vitro and the clinical response to drug therapy is often misleading. This discrepancy has been attributed to the in vivo reality, where the pathogen is exposed to diurnal fluctuating drug levels [11]. Moreover, as in our case, where the infection was compartmentalized within areas of subcutaneous inflammation, it is unclear as to how much of the drug actually penetrates the zone of infection and reaches the infecting fungus. Relapses following drug therapy are frequently reported $[8,9,11]$, requiring patients to undergo repetitive long-term antimycotic therapy, which often leads to off-target toxicity such as hepatotoxicity [9]. Interestingly, itraconazole therapy has been associated with congestive heart failure in a small subset of human patients [12,13] and carries a "black box warning" against administration to patients with ventricular dysfunction such as congestive heart failure due to negative inotropic activity (Sporonox, Janssen Pharmaceutica N.V., Olen Belgium). It is possible that the areas of fibrosis in this cat's heart were subclinical but predisposed the patient to the development of heart failure and subsequent bicavitary effusions after administration of itraconazole, although this cannot be confirmed in this case.

The European Advisory Board on Cat Diseases recommends aggressive surgical excision of local lesions prior to drug treatment when dealing with subcutaneous phaeohyphomycosis [7]. However, in a previous case report involving surgery in combination with long-term ketoconazole and fluconazole therapy for the treatment of E. attenuata feline phaeohyphomycosis, the treatment strategy ultimately was ineffective [8]. In this current case report, itraconazole treatment following surgery, at a dosage rate of $10 \mathrm{mg} / \mathrm{kg}$ orally once daily, also did not resolve the infection, although sensitivity of E. attenuata to itraconazole in vitro has been previously reported [5].

Conflict of interest The authors declare that they have no conflict of interest.

\section{References}

1. Seyedmousavi S, Netea MG, Mouton JW, Melchers WJG, Verweij PE, de Hoog GS. Black yeasts and their filamentous relatives: principles of pathogenesis and host defense. Clin Microbiol Rev. 2014;27:527-42.

2. de Hoog GS, Vicente VA, Najafzadeh MJ, Harrak MJ, Badali H, Seyedmousavi S. Waterborne Exophiala species causing disease in cold-blooded animals. Persoonia Mol Phylogeny Evol Fungi. 2011;27:46-72.

3. Domsch KH, Gams W, Anderson TH. Compendium of soil fungi. 2nd ed. Eching: IHW-Verlag; 2007.

4. Iwatsu T, Udagawa S, Takase T. A new species of Exophiala recovered from drinking water. Mycotaxon. 1991;41:321-8.

5. Zeng JS, Sutton DA, Fothergill AW, Rinaldi MG, Harrak MJ, de Hoog GS. Spectrum of clinically relevant Exophiala species in the United States. J Clin Microbiol. 2007;45:3713-20.

6. Vitale RG, de Hoog GS. Molecular diversity, new species and antifungal susceptibilities in the Exophiala spinifera clade. Med Mycol. 2002;40:545-56.

7. Lloret A, Hartmann K, Grazia Pennisi M, Ferrer L, Addie D, Belák S, Boucraut-Baralon C, Egberink H, Frymus T, Gruffydd-Jones T, Hosie MJ, Lutz H, Marsilio F, Möstl K, Radford AD, Thiry E, Truyen U, Horzinek MC. Rare opportunistic mycoses in cats phaeohyphomycosis and hyalohyphomycosis $\mathrm{ABCD}$ guidelines on prevention and management. J Feline Med Surg. 2013;15:628-30.

8. Chermette R, Ferreiro L, De Bievre C, Camadro JP, Mialot M, Vauzelle P. Exophiala spinifera nasal infection in a cat and a literature review of feline phaeohyphomycoses. J Mycol Med. 1997;7:149-58.

9. Kettlewell P, McGinnis MR, Wilkinson GT. Phaeohyphomycosis caused by Exophiala spinfera in two cats. J Med Vet Mycol. 1989;27:257-64. 
10. Nuttall W, Woodgyer A, Butler S. Phaeohyphomycosis caused by Exophiala jeanselmei in a domestic cat. NZ Vet J. 1990;38:128.

11. Vitale RG, De Hoog GS, Verweij PE. In vitro activity of amphotericin B, itraconazole, terbinafine and 5-fluocytosine against Exophiala spinifera and evaluation of post-antifungal effects. Med Mycol. 2003;41:301-7.
12. Ahmad SR, Singer SJ, Leissa BG. Congestive heart failure associated with itraconazole. Lancet. 2001;357:1766-7.

13. Vollenbroich R, Maeder MT, Weilenmann D. Congestive heart failure related to antifungal therapy with itraconazole. Int J Cardiol. 2014;172:e170-1. 